\title{
Estrutura de prática e liberdade de escolha na aprendizagem de habilidades motoras
}

\author{
Cinthya Walter ${ }^{1}$ \\ Flavio Henrique Bastos ${ }^{1}$ \\ Ulysses Okada Araujo ${ }^{1}$ \\ Jane A.O. Silva ${ }^{1,2}$ \\ Umberto Cesar Corrêa ${ }^{1}$
}

https://doi.org/10.5628/rpcd.08.03.337

\author{
${ }^{1}$ Laboratório de Comportamento Motor \\ Escola de Educação Física e Esporte \\ Universidade de São Paulo \\ São Paulo \\ Brasil \\ ${ }^{2}$ Universidade do Vale do Sapucaí \\ Pouso Alegre \\ Minas Gerais \\ Brasil
}

\section{RESUMO}

O objectivo do estudo foi investigar os efeitos de estruturas de prática com diferentes níveis de liberdade de escolha na aprendizagem de habilidades motoras. Realizou-se um experimento constituído de duas fases: estabilização e adaptação. Na fase de estabilização, as 120 crianças participantes foram distribuídas em seis grupos formados pela associação entre as estruturas de prática constante e constante-aleatória e diferentes tipos de liberdade de escolha - da sequencia ou de alguns componentes para formar uma sequência. Na fase de adaptação, os aprendizes foram testados em sua capacidade de se adaptar a uma modificação perceptivo-efetora da tarefa. O grupo que realizou a prática constante com liberdade na escolha de alguns componentes para formar a sequência foi mais preciso com relação ao erro absoluto do que os demais grupos, um dos mais precisos com relação ao erro constante e esteve entre os grupos mais consistentes (erro variável) na fase de adaptação. Esses resultados indicam que a estrutura de prática constante com liberdade na escolha dos componentes permitiu melhor adaptação à nova tarefa.

Palavras-chave: Estrutura de prática, liberdade de escolha, aprendizagem motora, processo adaptativo

\begin{abstract}
Practice schedule and freedom of choice in motor skill learning

The purpose of this study was to investigate the effects of practice schedules with different levels of freedom of choice on learning of motor skills. One experiment was carried out, consisting of two phases: stabilization and adaptation. In the stabilization phase 120 children were assigned into six groups formed by the association between constant and constant-random practice schedules and different kinds of freedom of choice - of the sequence or of some components to form a sequence. In the adaptation phase, the learners were tested on their capacity to adapt to a perceptual-motor task modification. The group that performed in a constant practice schedule with freedom in choice of some components was more accurate than the other groups regarding absolute error, it was one of the most accurate regarding constant error, and it was among the most consistent groups (variable error) in the adaptation phase. These results indicate that constant practice schedule with freedom in choice of components to form the sequence allowed better adaptation to the new task.
\end{abstract}

Key-words: Practice schedule, freedom of choice, motor learning, adaptive process 


\section{INTRODUÇÃO}

Entendendo os seres humanos como sistemas complexos adaptativos e, por conseguinte, a sua aprendizagem de habilidades motoras como um processo que envolve aumento de complexidade, Choshi(5) e Tani $(23,25,28,29)$ têm proposto um modelo de nãoequilíbrio de aprendizagem motora denominado Processo Adaptativo. Nesse modelo a aprendizagem de habilidades motoras é compreendida por duas fases: estabilização e adaptação. A fase de estabilização é aquela em que ocorre a busca por estabilidade funcional, na qual respostas inconsistentes ou erradas são gradualmente reduzidas por meio do feedback negativo até alcançar respostas consistentes e correctas, resultando na padronização espaço-temporal da habilidade. Quando isso acontece, infere-se que a estrutura da habilidade foi formada. Na fase de adaptação a estabilidade é desafiada por novas situações ou tarefas motoras. Essa fase se constitui pela modificação e reorganização da estrutura adquirida $(29,30)$. Em cada fase da vida a aprendizagem de habilidades motoras pode ocorrer em diferentes ambientes, sob condições diversas e em tempos distintos, mas ela se dá por meio de um mesmo factor: a prática.

Considerando que o movimento humano compreende não apenas um comportamento observável e mensurável, mas o produto final de um processo complexo que envolve a participação de vários mecanismos no sistema nervoso central(27), a prática pode ser entendida como um processo de exploração das várias possibilidades de solução de um problema motor, envolvendo, a cada tentativa, um esforço consciente de organização, execução, avaliação e modificação de acções motoras(27).

Por ser então um factor essencial para a aprendizagem motora, muitos estudos têm sido realizados no intuito de compreender seus efeitos. Esses estudos têm manipulado as estruturas de prática constante, variada e suas combinações $(1,6,7,8,9,10,11,24,30) \mathrm{e}$ grande parte das evidências tem apontado para a aprendizagem de habilidades motoras via a combinação das práticas constante e aleatória. A explicação atribuída a tais evidências remete-se à prática constante possibilitar a formação do padrão de interacção entre os componentes do sistema, ou seja, a forma- ção da estrutura da habilidade motora e, a prática aleatória, com a introdução de variação nos componentes, possibilitar sua diversificação( $(6,13)$.

Apesar disso, um aspecto que chama a atenção é que em todos esses trabalhos a prática foi totalmente determinada pelo pesquisador. Essa característica incita indagações no que diz respeito ao efeito da estruturação da prática que inclui alguma liberdade de escolha do aprendiz na aprendizagem de habilidades motoras, visto que esta variável tem sido um tema recorrente na literatura.

Vários estudos têm evidenciado efeitos positivos na aquisição de habilidades motoras quando é fornecido ao aprendiz algum tipo de liberdade de escolha sobre a sua prática $(2,3,4,16,17,23,31,33,34,35)$, mas dentre esses estudos apenas dois foram realizados sob um modelo de não-equilíbrio de aprendizagem $\operatorname{motora}^{(2,}$ 23) e não focalizaram os efeitos da estrutura de prática. Diante disso, o objectivo da presente pesquisa foi investigar os efeitos de diferentes estruturas de prática com liberdade de escolha na aprendizagem de habilidades motoras.

\section{MÉTODO}

Participaram desse experimento 120 crianças voluntárias de ambos os sexos, com média de idade de $10,8( \pm 1)$ anos, sem experiência prévia na tarefa utilizada. A participação no experimento foi condicionada ao consentimento livre e esclarecido assinado pelo responsável pela criança. O presente estudo foi aprovado pelo Comitê de Ética em Pesquisa da Escola de Educação Física e Esporte da Universidade de São Paulo (protocolo n²006/17).

Foi utilizado o aparelho de timing coincidente em tarefas complexas(12) que consiste de uma canaleta com 90 LEDs (light-emitting diodes), uma mesa de resposta, sensores metálicos, um dispositivo com cinco LEDs para fornecimento de feedback e um computador. A canaleta tem 200 centímetros de comprimento, 10 de largura e 10 de altura. O primeiro LED funciona como sinal de alerta. Após o acendimento desse LED, os próximos acendem e apagam sucessivamente simulando movimento. A mesa de resposta é de madeira, com 70 centímetros de comprimento, 90 de largura e seis de altura. Sobre a mesa foram dispostos seis sensores rectangulares medindo cinco 
centímetros de largura e 15 de comprimento (Figura 1). O dispositivo para fornecimento de feedback é composto de uma placa contendo cinco LEDs alinhados e, sobre cada um deles, da esquerda para a direita, as denominações: "muito antes", "antes", "certo", "depois", "muito depois". O dispositivo exibia "certo" quando a diferença entre o toque no sensor alvo e o acendimento do último LED da canaleta era menor ou igual a $30 \mathrm{~ms}$ (em módulo). As demais mensagens correspondiam a acréscimos/decréscimos de 30 ms em relação ao "certo".

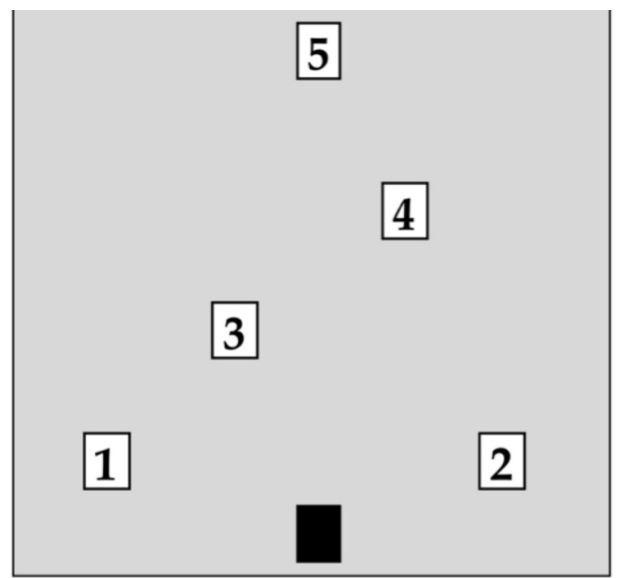

Figura 1. Esquema da disposição dos sensores na mesa de resposta do aparelho de timing coincidente em tarefas complexas.

A tarefa consistiu em tocar cinco sensores em uma sequência que variou de acordo com o grupo experimental, de forma que o último toque coincidisse com o acendimento do último LED (LED alvo).

Além dos cinco sensores a serem tocados havia um sensor que correspondia à posição inicial da mão do participante.

O experimento foi realizado em duas fases: estabilização e adaptação. Na fase de estabilização a prática foi conduzida até a obtenção de um desempenho critério - três tentativas consecutivas na faixa considerada como "certo" - mais 36 tentativas de acordo com a situação experimental de cada grupo, todas com a mesma velocidade do estímulo visual $(142,2$ $\mathrm{cm} / \mathrm{s}$ ) e com conhecimento de resultados. A utiliza- ção de um critério de desempenho foi adoptada para assegurar que os aprendizes atingissem um mesmo nível de desempenho nessa primeira fase do experimento, amenizando as diferenças individuais, além de servir como controle de possíveis experiências anteriores com tarefas similares.

Os participantes foram distribuídos em seis grupos experimentais: a) Co - prática constante - uma sequência determinada pelo experimentador (1-2-43-5) até o critério, mais 36 tentativas utilizando a mesma sequência; b) $\mathrm{Co}-\mathrm{Al}$ - prática constante seguida de variada aleatória - uma sequência determinada pelo experimentador até o critério (1-2-4-35), mais 36 tentativas utilizando as três sequências (1-2-4-3-5, 1-3-2-4-5 e 1-4-2-3-5) determinadas aleatoriamente pelo experimentador; c) $\mathrm{Co}(\mathrm{S})$ - prática constante com liberdade na escolha da sequência uma sequência escolhida pelo aprendiz, dentre três apresentadas (1-2-4-3-5, 1-3-2-4-5 ou 1-4-2-3-5), até o critério, mais 36 tentativas utilizando a mesma sequência escolhida; d) $\mathrm{Co}(\mathrm{S})$-L(S) - prática constante com liberdade na escolha da sequência seguida de liberdade na escolha da(s) sequência(s) - uma sequência escolhida pelo aprendiz, dentre três apresentadas (1-2-4-3-5, 1-3-2-4-5 ou 1-4-2-3-5), até o critério, mais 36 tentativas com liberdade de escolha entre as três sequências apresentadas (1-2-4-3-5, 13-2-4-5 e 1-4-2-3-5); e) Co(C) - prática constante com liberdade na escolha dos componentes para formar uma sequência - uma sequência formada pelo aprendiz, com liberdade na escolha dos componentes intermediários, uma vez que o primeiro e o último toque foram estabelecidos ( 1 e 5), até o critério, mais 36 tentativas utilizando a mesma sequência formada; f) $\mathrm{Co}(\mathrm{C})$-L(C) - prática constante com liberdade na escolha dos componentes para formar uma sequência seguida de liberdade na escolha dos componentes para formar uma ou mais sequências uma sequência formada pelo aprendiz, pela liberdade de escolha dos componentes intermediários, uma vez que o primeiro e o último toque foram estabelecidos ( 1 e 5), até o critério, mais 36 tentativas com a mesma liberdade de escolha, mas agora podendo variar a cada tentativa. O Quadro 1 ilustra os grupos experimentais na fase de estabilização. 
Quadro 1. Características dos seis grupos experimentais na fase de estabilização.

\begin{tabular}{|c|c|c|c|c|c|c|}
\hline \multirow[t]{3}{*}{ GRUPOS } & \multicolumn{6}{|c|}{ SEQUÊNCIA } \\
\hline & \multicolumn{3}{|c|}{ Até o critério } & \multicolumn{3}{|c|}{ Após o critério } \\
\hline & Determinada & Escolhida & Formada & Determinada & Escolhida & Formada \\
\hline Co & constante & & & constante & & \\
\hline Co-Al & constante & & & aleatória & & \\
\hline $\operatorname{Co}(\mathrm{S})$ & & constante & & & constante & \\
\hline Co(S)-L(S) & & constante & & & dade para $v$ & \\
\hline $\operatorname{Co}(\mathrm{C})$ & & & constante & & & constante \\
\hline Co(C)-L(C) & & & constante & & & rdade para variar \\
\hline
\end{tabular}

$\mathrm{Na}$ fase de adaptação foram executadas 36 tentativas em uma mesma situação para todos os grupos, sem conhecimento de resultados. Além da redução na velocidade do estímulo visual $(104,9 \mathrm{~cm} / \mathrm{s})$ foi solicitada a realização de uma nova sequência (1-5-2-34), envolvendo a modificação do sensor alvo. Essa modificação teve como objectivo garantir que a sequência fosse nova para todos os grupos.

Para verificar a compreensão da tarefa por parte da criança, era permitido que ela executasse sequências de toques até três vezes. No caso dos grupos com liberdade de escolha, após essa experimentação, a criança fazia a escolha da sequência a ser utilizada. Cada tentativa só era iniciada com o contacto da mão do participante no sensor inicial. Ao término da fase de estabilização, a criança era informada que teria que realizar uma sequência diferente das praticadas, era apresentada a nova sequência (1-5-2-3-4) e permitido que ela tocasse nos respectivos sensores, sem o estímulo visual. Logo que era informada de que nessa fase não iria mais receber feedback, a fase de adaptação era iniciada.

Os resultados foram analisados com relação a medidas que reflectem o desempenho no alcance da meta da tarefa (timing coincidente), por meio dos erros absoluto, variável e constante. Além das medidas relativas ao timing coincidente, foram utilizadas duas medidas complementares referentes ao padrão de movimento: erro de execução (estrutura macroscópica) e tempo total de movimento (estrutura microscópica) com relação à sua magnitude e variabilidade.
Os dados foram organizados em blocos de nove tentativas. Foram analisados o último bloco da fase de estabilização e todos os blocos da fase de adaptação. O último bloco de tentativas da fase de estabilização foi considerado como referência do desempenho dos grupos antes da introdução da modificação na tarefa (fase de adaptação). Primeiramente, os extremos foram substituídos pela mediana do seu grupo com o seu valor incluído e, posteriormente, foi calculada uma nova mediana. Apenas um extremo por sujeito foi substituído a fim de que a modificação dos dados não fosse superior a cinco por cento em cada bloco de tentativas.

Para a realização da análise inferencial foram testados os pressupostos para utilização da análise paramétrica, já que a natureza da variável dependente era intervalar. A independência das observações foi garantida e o pressuposto da normalidade foi assumido, visto a ANOVA ser um teste paramétrico robusto capaz de sobrepujar esse pressuposto quando o número de participantes por grupo é superior a quinze(15). Em seguida, foi testado o pressuposto da homogeneidade de variância e o teste de Levene indicou que as amostras não tinham homogeneidade. Contudo, as variâncias amostrais podem diferir como resultado da amostragem e o teste F não é invalidado por diferenças moderadas entre as variâncias amostrais ${ }^{(19,21)}$. Conforme Vincent ${ }^{(32)}$, como regra geral, a maior variância de um grupo não deve ser mais do que duas vezes a do grupo menor. Então, as variâncias de cada grupo foram calculadas e os resultados indicaram que as variâncias quase 


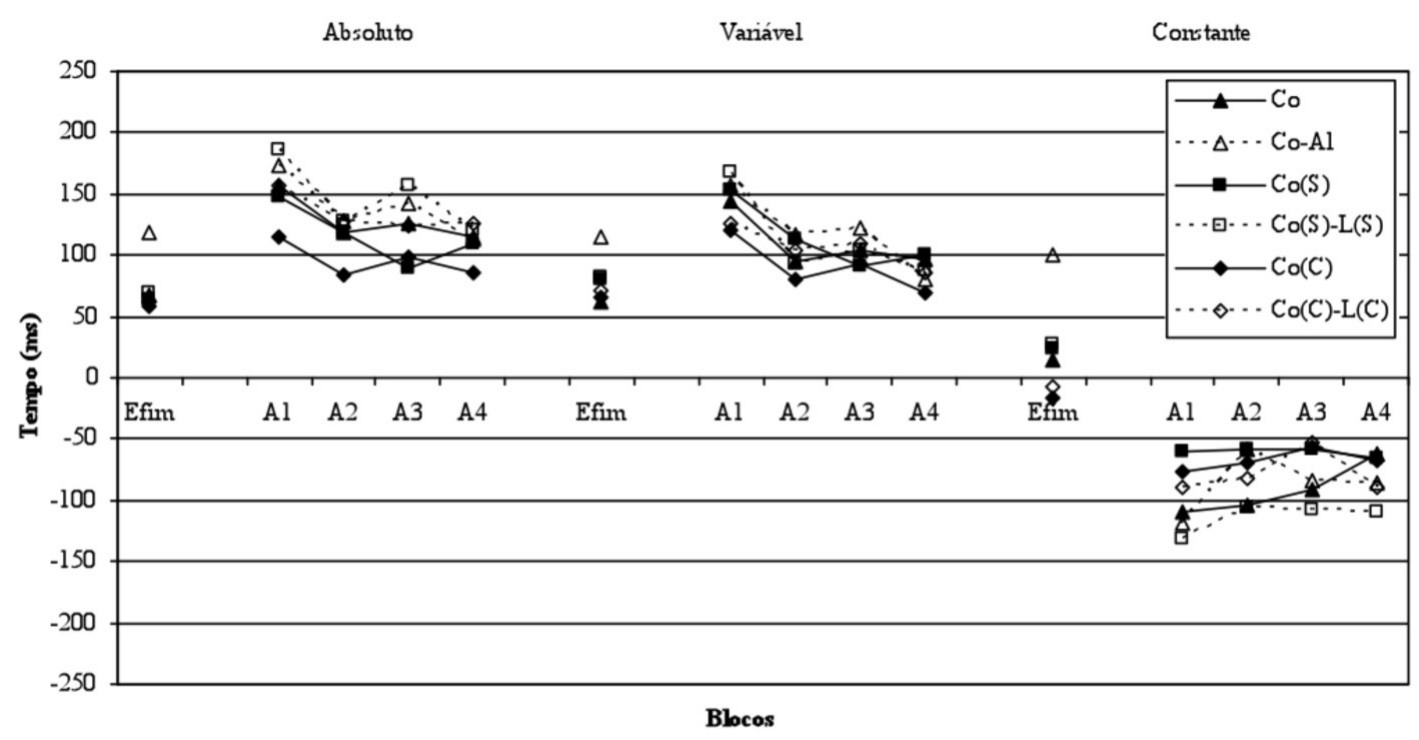

Figura 2. Medianas dos erros absoluto, variável e constante ( $\mathrm{ms}$ ], no último bloco de tentativas da fase de estabilização (Efim) e nos quatro blocos da fase de adaptação (A1, A2, A3 e A4), dos seis grupos experimentais [Co, Co-Al, Co(S), Co(S)-L(S), Co(C) e Co(C)-L(C)].

sempre foram superiores a duas vezes as das menores, de forma que esse pressuposto não foi atendido. Assim, foram utilizados testes não-paramétricos de Friedman para cada grupo, a fim de verificar as diferenças entre os blocos de tentativas e o teste de Kruskal-Wallis em cada bloco para verificar se havia diferenças entre os grupos. Posteriormente, foram realizados testes de post hoc sugeridos por Siegel e Castellan(22) a fim de localizar as diferenças encontradas tanto entre os blocos de tentativas ( $\mathrm{p}$ ajustado $<0,000556$ ) como entre os grupos ( $\mathrm{p}$ ajustado $<0,000238)$.

\section{RESULTADOS}

Os resultados das medidas de desempenho relacionadas ao alcance da meta da tarefa - erros absoluto, variável e constante - são ilustrados na Figura 2.

$\mathrm{Na}$ análise entre grupos para a medida de erro absoluto, o teste Kruskal-Wallis encontrou diferenças no último bloco de tentativas da fase de estabilização $\left[\chi^{2}(n=119, g l=5)=25,90, p=0,0001\right]$, no primeiro $\left[\chi^{2}(n=118, g l=5)=17,55, p=0,0036\right]$, no segundo $\left[\chi^{2}(\mathrm{n}=118, \mathrm{gl}=5)=15,27, \mathrm{p}=0,0092\right]$ e no terceiro $\left[\chi^{2}(n=120, g l=5)=16,68, p=0,0051\right]$ bloco da fase de adaptação. O post hoc associado à análise descritiva apontou que o grupo $\mathrm{Co}(\mathrm{C})$ foi mais preciso do que os grupos $\mathrm{Co}-\mathrm{Al}$ e $\mathrm{Co}(\mathrm{S})-\mathrm{L}(\mathrm{S})$ no último bloco de tentativas da fase de estabilização e no primeiro bloco da fase de adaptação; no segundo bloco de tentativas da fase de adaptação foi mais preciso do que todos os outros grupos; e no terceiro bloco foi mais preciso do que os grupos Co, Co-Al, $\mathrm{Co}(\mathrm{S})-\mathrm{L}(\mathrm{S})$ e $\mathrm{Co}(\mathrm{C})-\mathrm{L}(\mathrm{C})$.

Na comparação entre blocos o teste de Friedman encontrou diferença em todos os grupos: Co $\left[\chi^{2}(\mathrm{n}=19, \mathrm{gl}=4)=34,82105, \mathrm{p}<0,01\right]$; Co-Al $\left[\chi^{2}(\mathrm{n}=19, \mathrm{gl}=4)=20,12632, \mathrm{p}<0,01\right] ; \mathrm{Co}(\mathrm{S})\left[\chi^{2}\right.$ $(\mathrm{n}=20, \mathrm{gl}=4)=33,16000, \mathrm{p}<0,01] ; \mathrm{Co}(\mathrm{S})-\mathrm{L}(\mathrm{S})$ $\left[\chi^{2}(\mathrm{n}=20, \mathrm{gl}=4)=50,20000, \mathrm{p}<0,01\right] ; \mathrm{Co}(\mathrm{C})$ $\left[\chi^{2}(\mathrm{n}=20, \mathrm{gl}=4)=31,20000, \mathrm{p}<0,01\right]$ e $\mathrm{Co}(\mathrm{C})-\mathrm{L}(\mathrm{C})$ $\left[\chi^{2}(n=18, g l=4)=36,48889, p<0,01\right]$. O post hoc localizou a diferença para todos os grupos, com exceção do Co-Al, entre o último bloco de tentativas da fase de estabilização e o primeiro bloco da fase de adaptação. Assim, concernente ao erro absoluto, em todos esses grupos que tiveram diferenças entre o último bloco de tentativas da fase de estabilização e o primeiro bloco da fase de adaptação, a mudança na 
tarefa provocou piora no desempenho em termos de precisão. O fato do grupo $\mathrm{Co}-\mathrm{Al}$ não ter apresentado diferenças entre esses blocos de tentativas pode ser explicado pelo erro absoluto desse grupo, no último bloco da fase estabilização, ter se apresentado superior ao dos demais grupos.

No tocante ao erro variável, o teste Kruskal-Wallis encontrou diferença no último bloco de tentativas da fase de estabilização $\left[\chi^{2}(\mathrm{n}=119, \mathrm{gl}=5)=22,34\right.$, $\mathrm{p}=0,0005]$ e o post hoc associado à análise descritiva apontou que os grupos $\mathrm{Co}, \mathrm{Co}(\mathrm{C})$ e $\mathrm{Co}(\mathrm{C})-\mathrm{L}(\mathrm{C})$ foram mais consistentes do que os grupos $\mathrm{Co}-\mathrm{Al}$ e $\mathrm{Co}(\mathrm{S})-\mathrm{L}(\mathrm{S})$. Na fase de adaptação não foram encontradas diferenças entre os grupos.

Com relação às comparações intra-grupo, o teste de Friedman indicou diferença entre os blocos nos grupos $\mathrm{Co}(\mathrm{S})-\mathrm{L}(\mathrm{S})\left[\chi^{2}(\mathrm{n}=20, \mathrm{gl}=4)=20,48000\right.$, $\mathrm{p}<0,00040]$ e $\mathrm{Co}(\mathrm{C})\left[\chi^{2}(\mathrm{n}=20, \mathrm{gl}=4)=16,48000\right.$, $\mathrm{p}<0,00244]$. O post hoc associado à análise descritiva mostrou que na fase de adaptação, os grupos $\mathrm{Co}(\mathrm{S})$ $\mathrm{L}(\mathrm{S})$ e $\mathrm{Co}(\mathrm{C})$ aumentaram a sua consistência do primeiro para o último bloco de tentativas dessa fase. Referente ao erro constante, o teste Kruskal-Wallis encontrou diferença no último bloco de tentativas da fase de estabilização $\left[\chi^{2}(\mathrm{n}=120, \mathrm{gl}=5)=21,69\right.$, $\mathrm{p}=0,0006]$ e no primeiro bloco de tentativas da fase de adaptação $\left[\chi^{2}(\mathrm{n}=120, \mathrm{gl}=5)=12,21, \mathrm{p}=0,0319\right]$. $\mathrm{O}$ post hoc associado à análise descritiva apontou que o grupo Co esteve mais próximo ao acerto do que os demais grupos no último bloco de tentativas da fase de estabilização e, o grupo $\mathrm{Co}(\mathrm{S})$ adiantou menos do que os grupos $\mathrm{Co}-\mathrm{Al}, \mathrm{Co}(\mathrm{S})-\mathrm{L}(\mathrm{S}), \mathrm{Co}(\mathrm{C})$ e $\mathrm{Co}(\mathrm{C})$ $\mathrm{L}(\mathrm{C})$ no primeiro bloco de tentativas da fase de adaptação.

O teste de Friedman mostrou que todos os grupos apresentaram diferenças entre os blocos de tentativas: $\operatorname{Co}\left[\chi^{2}(\mathrm{n}=20, \mathrm{gl}=4)=22,24000, \mathrm{p}<0,00018\right]$, Co-Al $\left[\chi^{2}(\mathrm{n}=20, \mathrm{gl}=4)=39,32000, \mathrm{p}<0,00000\right]$, $\mathrm{Co}(\mathrm{S})\left[\chi^{2}(\mathrm{n}=20, \mathrm{gl}=4)=31,24000, \mathrm{p}<0,00000\right]$, $\operatorname{Co}(\mathrm{S})-\mathrm{L}(\mathrm{S})\left[\chi^{2}(\mathrm{n}=20, \mathrm{gl}=4)=42,56000\right.$, $\mathrm{p}<0,00000], \operatorname{Co}(\mathrm{C})\left[\chi^{2}(\mathrm{n}=20, \mathrm{gl}=4)=27,32000\right.$, $\mathrm{p}<0,00002]$ e $\mathrm{Co}(\mathrm{C})-\mathrm{L}(\mathrm{C})\left[\chi^{2}(\mathrm{n}=20\right.$, $\mathrm{gl}=4)=11,48000, \mathrm{p}<0,02167]$. O post hoc apontou que todos os grupos foram diferentes do último bloco de tentativas da fase de estabilização para o primeiro bloco da fase de adaptação. Assim, concernente ao erro constante, a modificação na tarefa pro- vocou mudança na direcção do desempenho, mais especificamente, a análise descritiva aponta que todos os grupos passaram a adiantar ou adiantaram mais a sua resposta no primeiro bloco de tentativas da fase de adaptação.

Os resultados relativos às medidas do padrão de movimento (erro de execução e tempo de movimento) são ilustrados nas figuras 3 e 4 .

Com relação à medida de erro de execução, que mostra quantas tentativas no bloco de nove foram executadas de forma incompleta ou na sequência incorrecta, o teste Kruskal-Wallis encontrou diferença entre os grupos apenas no último bloco de tentativas da fase de estabilização $\left[\chi^{2}(n=120\right.$, $\mathrm{gl}=5)=33,29, \mathrm{p}=0,0000]$. O post hoc associado à análise descritiva indicou que os grupos $\mathrm{Co}(\mathrm{C})$, $\mathrm{Co}(\mathrm{C})-\mathrm{L}(\mathrm{C})$ e $\mathrm{Co}(\mathrm{S})-\mathrm{L}(\mathrm{S})$ tiveram menos erros de execução do que o grupo Co-Al no último bloco de tentativas da fase de estabilização.

Por sua vez, o teste de Friedman indicou que o grupo $\mathrm{Co}-\mathrm{Al}$ apresentou diferenças entre os blocos de tentativas $\left[\chi^{2}(n=20, g l=4)=27,00, p<0,00002\right]$. $\mathrm{O}$ post hoc associado à análise descritiva apontou uma redução dos erros de execução do último bloco de tentativas da fase de estabilização para o terceiro bloco da fase de adaptação.

A medida de tempo total de movimento se refere ao tempo gasto entre o início do movimento e o toque no último sensor. Ela foi calculada em termos de magnitude e variabilidade.

No que diz respeito à magnitude, na análise entre grupos, o teste Kruskal-Wallis encontrou diferença apenas no último bloco de tentativas da fase de estabilização $\left[\chi^{2}(\mathrm{n}=120, \mathrm{gl}=5)=20,25, \mathrm{p}<0,0011\right]$. $\mathrm{O}$ post hoc associado à análise descritiva indicou que o tempo total de movimento do grupo Co-Al foi mais alto do que os dos grupos $\mathrm{Co}(\mathrm{C})$ e $\mathrm{Co}(\mathrm{C})-\mathrm{L}(\mathrm{C})$. $\mathrm{Na}$ fase de adaptação, não foram encontradas diferenças entre os grupos, indicando que eles usaram tempos totais de movimento semelhantes.

Nas comparações intra-grupo relativas à magnitude do tempo de movimento, o teste de Friedman indicou que todos os grupos apresentaram diferenças entre os blocos de tentativas: Co $\left[\chi^{2}(\mathrm{n}=20\right.$, $\mathrm{gl}=4)=43,32, \mathrm{p}<0,00000]$, Co-Al $\left[\chi^{2}(\mathrm{n}=20\right.$, $\mathrm{gl}=4)=43,56, \mathrm{p}<0,00000], \mathrm{Co}(\mathrm{S})\left[\chi^{2}(\mathrm{n}=20\right.$, $\mathrm{gl}=4)=41,80, \mathrm{p}<0,00000], \mathrm{Co}(\mathrm{S})-\mathrm{L}(\mathrm{S})\left[\chi^{2}(\mathrm{n}=20\right.$, 


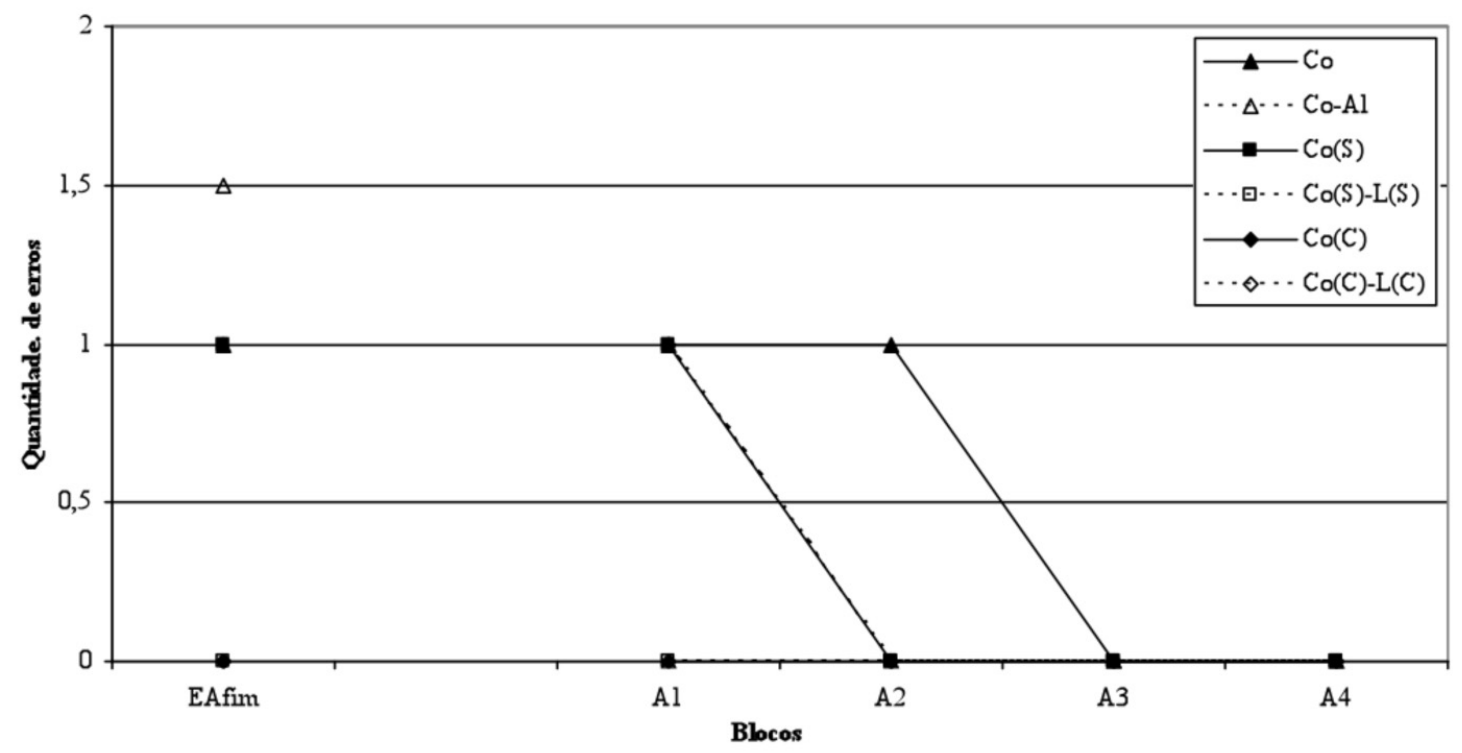

Figura 3. Medianas do erro de execução, em número de tentativas, dos seis grupos experimentais [Co, $\operatorname{Co-Al,} \operatorname{Co}(S), \operatorname{Co}(S)-L[S], \operatorname{Co}(C)$ e $\operatorname{Co}[C]-L(C)]$, no último bloco de tentativas da fase de estabilização (Efim) e nos quatro blocos de tentativas da fase de adaptação (A1, A2, A3 e A4].

$\mathrm{gl}=4)=45,92, \mathrm{p}<0,00000], \mathrm{Co}(\mathrm{C})\left[\chi^{2}(\mathrm{n}=20\right.$ $\mathrm{gl}=4)=44,04, \mathrm{p}<0,00000]$ e $\mathrm{Co}(\mathrm{C})-\mathrm{L}(\mathrm{C})\left[\chi^{2}(\mathrm{n}=20\right.$, $\mathrm{gl}=4)=42,72, \mathrm{p}<0,00000]$. O post hoc associado à análise descritiva apontou que todos os grupos apresentaram tempo total de movimento maior em todos os blocos de tentativas da fase de adaptação quando comparados com o tempo total de movimento do final da fase de estabilização, com excepção do grupo Co-Al que só não apresentou aumento do fim da fase de estabilização para o primeiro bloco da fase de adaptação.

Relativo à variabilidade do tempo total de movimento, o teste Kruskal-Wallis não encontrou diferença entre os grupos em nenhum dos blocos de tentativas, indicando que as estratégias de toques utilizadas foram semelhantes entre os grupos. Nas comparações intragrupo, o teste de Friedman indicou que houve diferença entre os blocos de tentativas nos grupos Co-Al $\left[\chi^{2}(\mathrm{n}=20, \mathrm{gl}=4)=23,32000\right.$, $\mathrm{p}<0,00011], \mathrm{Co}(\mathrm{S})\left[\chi^{2}(\mathrm{n}=20, \mathrm{gl}=4)=17,08000\right.$, $\mathrm{p}<0,00187], \mathrm{Co}(\mathrm{S})-\mathrm{L}(\mathrm{S})\left[\chi^{2}(\mathrm{n}=20\right.$, $\mathrm{gl}=4)=18,04000, \mathrm{p}<0,00121]$ e $\mathrm{Co}(\mathrm{C})\left[\chi^{2}(\mathrm{n}=20, \mathrm{gl}\right.$ $=4)=17,12000, \mathrm{p}<0,00183]$. O post hoc associado à análise descritiva apontou que o grupo Co-Al reduziu a sua variabilidade do fim da fase de estabilização e do começo da fase de adaptação para o último bloco de tentativas da fase de adaptação. Por sua vez, os grupos $\mathrm{Co}(\mathrm{S})-\mathrm{L}(\mathrm{S})$ e $\mathrm{Co}(\mathrm{C})$ reduziram a sua variabilidade do primeiro para o último bloco de tentativas da fase de adaptação.

\section{DISCUSSÃO}

Os resultados mostraram que a prática constante com liberdade na escolha dos componentes foi a que proporcionou melhores resultados na adaptação a uma nova tarefa. Isso porque o grupo submetido a esse tipo de prática foi mais preciso com relação ao erro absoluto que os demais grupos, um dos mais precisos com relação ao erro constante e esteve entre os grupos mais consistentes (erro variável) na fase de adaptação. Além disso, foi um dos dois grupos que reduziram a variabilidade do tempo total de movimento do primeiro para o último bloco de tentativas da fase de adaptação.

Até então, no que se refere à aquisição de habilidades motoras, as evidências sugeriam que primeira- 

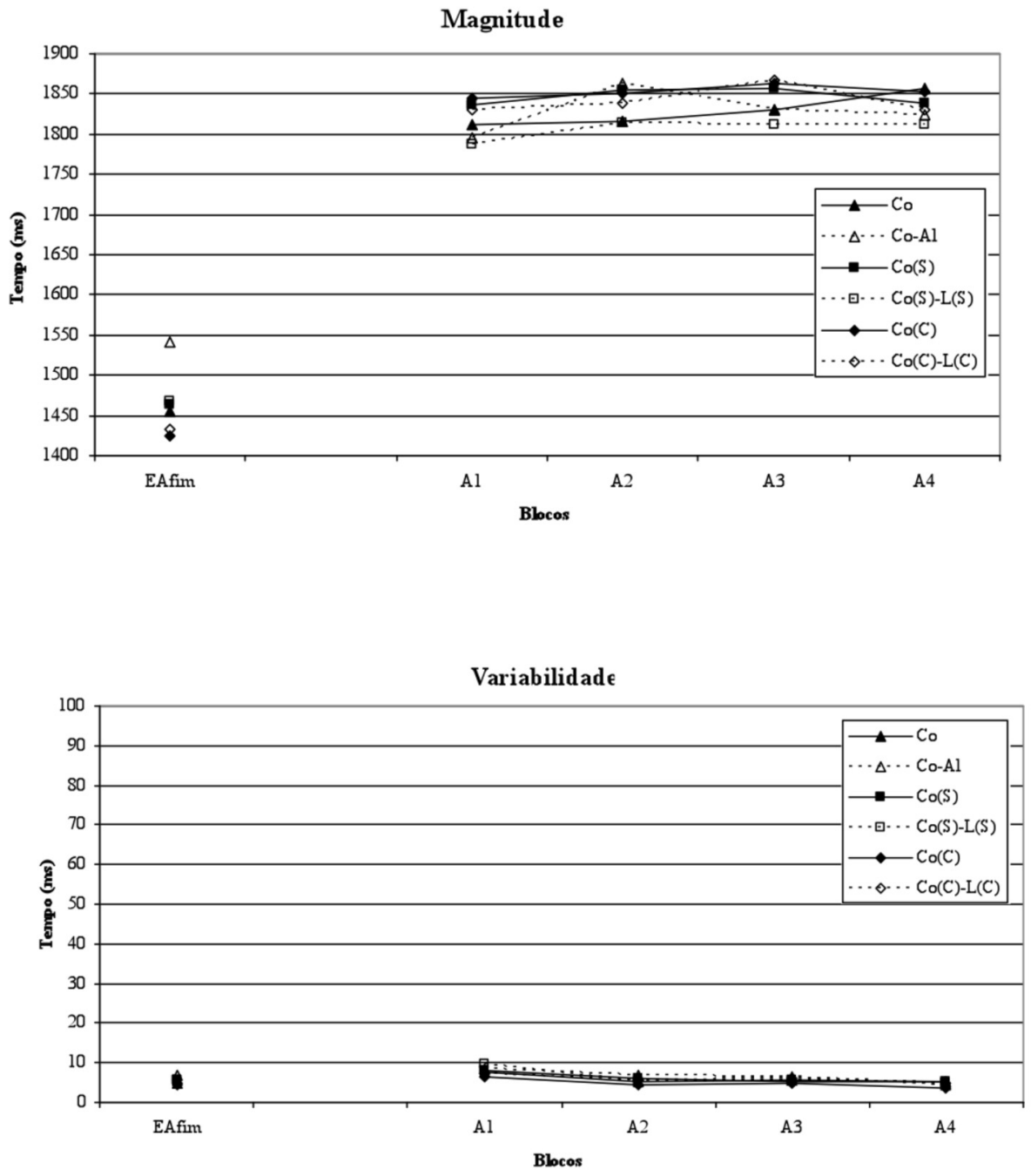

Figura 4. Medianas do tempo total de movimento (magnitude) e da sua variabilidade (ms), no último bloco de tentativas da fase de estabilização (Efim) e nos quatro blocos de tentativas da fase de adaptação $[A 1, A 2, A 3$ e A4] dos seis grupos experimentais [Co, Co-Al, $C o[S], C o[S]-L(S), C o(C)$ e $C o(C)-L(C)]$.

mente haveria necessidade da prática constante para se formar a estrutura da habilidade e, posteriormente, da prática aleatória para promover sua diversificação, possibilitando flexibilidade à habilidade, ou seja, capacidade de adaptação(13). Entretanto, a estrutura de prática constante (tradicionalmente caracterizada pela repetição da informação, estabilidade, consistência, ordem e precisão) além de permitir o alcance da estabilidade num conjunto de soluções apropriadas(27) pode possibilitar a formação de um padrão de interação entre os componentes do sistema com flexibilidade suficiente para se adaptar a novas situações $(8,13)$. Os resultados do presente estudo demonstram que a efectividade da estrutura de prática constante para a aprendizagem motora é aumentada quando se oferece ao aprendiz liberdade na escolha 
da resposta motora, especificamente no que se refere à escolha de componentes intermediários que formam a sequência. Esse resultado dá suporte à hipótese de que para se adquirir padrões flexíveis de movimento que melhor se adaptam às novas situações seja preciso permitir ao aprendiz um certo grau de liberdade na escolha das respostas durante o processo de estabilização(23).

Uma possível explicação para esse resultado é que a estrutura de prática constante com liberdade na escolha dos componentes intermediários permitiu que o aprendiz seleccionasse uma sequência mais confortável para a sua prática - sendo o termo conforto entendido como uma condição em que o sistema de controle opera satisfatoriamente e que essa condição é gerada quando o sistema desfruta de segurança e pode actuar de maneira económica(14). Dentre as cinco sequências escolhidas pelos aprendizes, duas foram mais utilizadas: 1-2-3-4-5 (nove participantes) e 1-3-2-4-5 (oito participantes). A posição dos sensores sobre a mesa de resposta permite notar que as sequências mais escolhidas continham reversões mais curtas e em menor quantidade dentre as possíveis. Nesse sentido, a referida estrutura de prática possibilitou que o sistema adquirisse maior disponibilidade de recursos, ou redundância no sistema, do que as demais estruturas de prática com e sem liberdade de escolha do aprendiz. Assim, os resultados do presente estudo corroboram a literatura, uma vez que a redundância alcançada na estabilização foi fundamental para a adaptação(25).

Nesse estudo os grupos foram manipulados em relação a nenhum ou a algum controle em dois aspectos: estrutura de prática a ser realizada e resposta motora. O grupo que melhor se adaptou teve a estrutura de prática constante determinada pelo experimentador durante toda fase de estabilização. Entretanto, com relação à resposta motora, esse grupo teve o primeiro e o último toque determinados pelo experimentador, mas pôde formar a sequência. Dessa forma, foi oferecido aos aprendizes um referencial para a resposta motora (início e fim determinados), sem que fosse eliminada a possibilidade de variabilidade (liberdade para a formação da sequência). Isso fortalece a afirmação de que proporcionar liberdade na escolha de alternativas encoraja os aprendizes a explorarem suas potencialidades de movimento, pois são dadas infor- mações sobre a macroestrutura do mesmo apenas como referencial orientador dessa exploração(26). Outra afirmação que também é fortalecida com esses resultados é a de que em habilidades motoras estão presentes regras fixas que resultam na estabilidade macroscópica e estratégias flexíveis responsáveis pela variabilidade microscópica ${ }^{(18,20)}$.

No que concerne à existência de um grau adequado de liberdade de escolha do aprendiz, os resultados desse estudo apontam que as estruturas de prática constante com liberdade na escolha da sequência seguida de liberdade na escolha da(s) sequência(s); constante seguida de variada aleatória e constante foram, nessa ordem, as que levaram a uma maior dificuldade na adaptação à modificação da tarefa.

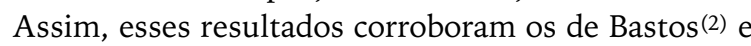
Tani(23) no sentido da ausência de liberdade de escolha (aprendizagem totalmente dirigida) enfatizar somente o aspecto invariável da habilidade, contribuindo para a formação de padrões de movimento estereotipados, de difícil adaptação e de não ser qualquer grau de liberdade que proporciona benefícios à aprendizagem motora.

Com base nos resultados obtidos pode-se concluir que o grupo que teve a prática constante com liberdade na escolha dos componentes intermediários para formar uma sequência foi aquele que alcançou melhores resultados na adaptação a uma nova tarefa (com modificação perceptivo-efetora), sendo assim, o grupo mais eficiente no processo adaptativo em aprendizagem motora. Considerando as delimitações da presente pesquisa e os resultados obtidos, percebe-se a importância de dar continuidade à investigação dessas variáveis, ou seja, de buscar mais conhecimentos sobre os efeitos de diferentes estruturas de prática com liberdade de escolha na aprendizagem de habilidades motoras.

\section{AGRADECIMENTOS}

Agradecemos ao CNPq - Brasil o apoio concedido durante a realização desse trabalho.

\section{CORRESPONDÊNCIA}

\section{Cinthya Walter}

Av. Prof. Mello Moraes, $\mathrm{n}^{\circ} 65$

CEP: 05508-900 - São Paulo - SP - BRASIL

E-mail: cinthyaw@usp.br 


\section{REFERÊNCIAS BIBLIOGRÁFICAS}

1. Barros JAC (2006). Estrutura de prática e processo adaptativo em aprendizagem motora: efeitos da especificidade da tarefa. Dissertação (Mestrado) - Escola de Educação Física e Esporte da Universidade de São Paulo, São Paulo.

2. Bastos FH (2007). Efeito do grau de liberdade na escolha da resposta no processo adaptativo em aprendizagem motora. Dissertação (Mestrado) - Escola de Educação Física e Esporte da Universidade de São Paulo, São Paulo.

3. Bund A, Wiemeyer J (2004). Self-controlled learning of a complex motor skill: Effects of the learner's preferences on performance and self-efficacy. J Hum Mov Studies, 47: 215236.

4. Chiviacowsky S, Wulf G (2002). Self-controlled feedback: does it enhance learning because performers get feedback when they need it? Res Q Exerc Sport, 73 (4): 408-415.

5. Choshi K (2000) Aprendizagem motora como um problema mal-definido. Rev Paul Educ Fís, supl. 3: 16-23.

6. Corrêa UC (2001). Estrutura de prática e o processo adaptativo na aquisição de habilidades motoras. Tese (Doutorado) Escola de Educação Física e Esporte da Universidade de São Paulo, São Paulo.

7. Corrêa UC, Barros JAC, Gonçalves LA, Massigli M, Souza Jr OP (2004). Constant-random practice and adaptive process in motor learning: effect of different quantities of constant practice on motor skill acquisition. J Sport Exerc Psychol 26: S59.

8. Corrêa UC, Benda RN, Meira Júnior CM, Tani G (2003). Practice schedule and adaptative process in the acquisition of a manual force control task. J Hum Mov Studies 44: 121138.

9. Corrêa UC, Benda RN, Tani G (2001). Estrutura de prática e o processo adaptativo na aquisição do arremesso de dardo de salão. Rev Bras Cienc Esporte 22 (2) 69-83.

10. Corrêa UC, Gonçalves LA, Barros JAC, Massigli M (2006). Prática constante-aleatória e aprendizagem motora: efeitos da quantidade de prática constante e da manipulação de exigências da tarefa. Brazilian Journal of Motor Behavior 1: 41-52.

11. Corrêa UC, Massigli M, Gonçalves LA, Barros JAC (no prelo). Constant-random practice and adaptive process in motor learning: effects of different amounts of constant practice. J Hum Mov Studies.

12. Corrêa UC, Tani G (2004). Aparelho de timing coincidente em tarefas complexas. PI $n^{\circ}$ 0.4.04.433-4 de 03/08/2004. Revista da Propriedade Industrial - RPI 1763: 178.

13. Corrêa UC, Tani G (2005). Estrutura de prática e processo adaptativo em aprendizagem motora: por uma nova abordagem da prática. In: TANI, G. (Ed). Comportamento motor: aprendizagem e desenvolvimento. Rio de Janeiro: Guanabara Koogan, p.141-161.

14. Gimenez R, Manoel EJ (2005). Comportamento Motor e deficiência: considerações para pesquisa e intervenção. In: TANI, G. (Ed). Comportamento motor: aprendizagem e desenvolvimento. Rio de Janeiro: Guanabara Koogan, p.314-327.

15. Green SB, Salkind NJ, Akey TM (2000). Using SPSS for Windows: analyzing and understanding data. 2 ed. New Jersey: Prentice Hall.

16. Janelle CM, Barba DA, Frehlich SG, Tennant LK, Cauraugh
JH (1997). Maximizing performance feedback effectiveness through videotape replay and a self-controlled learning environment. Res Q Exerc Sport 68 (4): 269-279.

17. Janelle CM, Kim J, Singer RN (1995). Subject-controlled performance feedback and learning of a closed motor skill. Perc Mot Skills 81 (2): 627-634.

18. Koestler A (1967). The ghost in the machine. London: Hutchinson.

19. Levin J, Fox JA (2004). Estatística para ciências humanas. 9 ed. São Paulo: Prentice Hall.

20. Manoel E de J, Connolly KJ (1995). Variability and the development of skilled actions. Int J Psychophysiol 19: 129147.

21. Pestana MH, Gageiro JN (2005). A análise de dados para ciências sociais: a complementaridade do SPSS. 4 ed. Lisboa: Edições Sílabo.

22. Siegel S, Castellan NJ (1988). Nomparametrics statistics. 2 ed. New York: Mc Graw-Hill Int.

23. Tani G (1982). Processo adaptativo na aprendizagem de uma habilidade perceptivo-motora. Tese (Doutorado) Universidade de Hiroshima, Hiroshima (Resumo).

24. Tani G (1989).Variabilidade de resposta e o processo adaptativo em aprendizagem motora. Tese (Livre Docência) - Escola de Educação Física, Universidade de São Paulo, São Paulo.

25. Tani G (1995). Hierarchical organization of human motor behaviour. Sheffield, University of Sheffield. (Unpublished Technical Report).

26. Tani G (1998). Liberdade e restrição do movimento no desenvolvimento motor da criança. In: Krebs, R.J.; Copetti, F. \& Beltrame, T.S. (Orgs). Discutindo o desenvolvimento infantil. Santa Maria: Palloti, p.39-62.

27. Tani G (1999). Criança e movimento: o conceito de prática na aquisição de habilidades motoras. In: Krebs, R.J.; Copetti, F.; Beltrame, T.S. \& Ustra, M. (Orgs). Perspectivas para o desenvolvimento infantil. Santa Maria: Edições SIEC, p.57-64.

28. Tani G (2000). Processo adaptativo em aprendizagem motora: o papel da variabilidade. Rev Paul Educ Fís supl. 3: 55-61.

29. Tani G (2005). Processo adaptativo: uma concepção de aprendizagem motora além da estabilização. In: Tani, G. (Ed). Comportamento motor: aprendizagem e desenvolvimento. Rio de Janeiro: Guanabara Koogan,. p.60-70.

30. Tani G, Bastos FC, Castro IJ, Jesus JF, Sacay RC, Passos SCE (1992) Variabilidade de resposta e processo adaptativo em aprendizagem motora. Rev Paul Educ Fís 6 (1): 1625.

31. Titzer R, Shea JB, Romack J (1993) The effect of learner control on the acquisition and retention of a motor task. $J$ Sport Exerc Psychol,15: S84.

32. Vincent WJ (1999). Statistics in Kinesiology. 2 ed. Champaign: Human Kinetics.

33. Wu W, Magill R (2004) To dictate or not: the exploration of a self-regulated practice schedule. J Sport Exerc Psychol 26: S202.

34. Wulf G, Raupach M, Pfeiffer F (2005). Self-controlled observational practice enhances learning. Res Q Exerc Sport 76: 107-111.

35. Wulf G, Toole T (1999). Physical assistance devices in complex motor skill learning: benefits of a self-controlled practice schedule. Res Q Exerc Sport 70 (3): 265-272. 